\title{
Oscillatory Solutions of Lossless Transmission Lines Terminated by Nonlinear Resistance and in Series Connected Inductance
}

\author{
Vasil G. Angelov \\ Department of Mathematics, University of Mining and Geology "St. I. Rilski”, Sofia, Bulgaria \\ Email: angelov@mgu.bg
}

How to cite this paper: Angelov, V.G. (2017) Oscillatory Solutions of Lossless Transmission Lines Terminated by Nonlinear Resistance and in Series Connected Inductance. Open Access Library Journal, 4: e3884. https://doi.org/10.4236/oalib.1103884

Received: August 14, 2017

Accepted: September 25, 2017

Published: September 28, 2017

Copyright (c) 2017 by author and Open Access Library Inc.

This work is licensed under the Creative Commons Attribution International License (CC BY 4.0).

http://creativecommons.org/licenses/by/4.0/ (c) (i) Open Access

\begin{abstract}
The present paper is devoted to the investigation of lossless transmission lines terminated by a nonlinear load with an interval of negative differential resistance and in series connected conductance. In contrast of almost all paper when $i=f(u)$, here the case $u=f(i)$ is considered. A general method of reducing the mixed problem to an initial value problem for neutral system on the boundary is presented. Sufficient conditions for the existence-uniqueness of an oscillatory solution are formulated. This is achieved by introducing an appropriate operator acting on suitable function space whose fixed point is an oscillatory solution of the initial value problem.
\end{abstract}

\section{Subject Areas}

Multimedia/Signal Processing

\section{Keywords}

Lossless Transmission Lines, Oscillatory Solution, Nonlinear Resistance, Linear Inductance, Fixed Point Method

\section{Introduction}

It is well known that all electronic circuits are nonlinear and the linear assumption is only approximation (cf. [1]-[9]). Here a lossless transmission line terminated by a nonlinear resistive element is considered. In view of [1], every resistive element with an interval of negative differential resistance falls into one of the following three groups: 1) The current is a single-valued function of the voltage. Such an element is called a nonlinear conductance. For more complete description of the conductance it is assumed to be shunted by parasitic parallel con- 
nected capacitance; 2) The voltage is a single-valued function of the current. Such an element is called a nonlinear resistance. In this case to obtain complete description of the resistance one should include in series parasitic inductance; 3 ) The characteristic $V-I$ is a multi-valued function of both current and voltage. In almost all papers only the first case is investigated [2]-[17]. The primary goal of the present paper is to investigate the second case.

Let us note that the above classification corresponds to Ohm's law $u=R i$. This means that the relation $u=f(i)$ should be called nonlinear resistance, while $i=f(u),(i=G u)$-nonlinear conductance [1]. If we try to study the second case in a usual way we have to solve the equation $u=f(i)$ with respect to $i$, that is, $i=f^{-1}(u)$. But, in general, the inverse function is multi-valued one which leads to multi-valued boundary conditions and hence to multi-valued differential equations with retarded arguments or differential inclusions. Many examples are given in [1]-[18]. For instance (cf. [19]), in lightly doped varactors, electrons may approach saturated drift velocity in the resistive epilayer. This phenomenon increases the incremental resistance in a nonlinear manner. One approach to modeling saturation is by the function $u=R_{s 0}\left(i+\alpha i^{7}\right)$.

Figure 1 shows a lossless transmission line terminated by nonlinear load and in series connected linear inductance where $J_{0}(t)$ is a current source function, $G_{0}$-the conductance of the source and $L_{1}$-linear inductance. The nonlinear load has an $I-V$ characteristic of the type $u=f(i)$.

It is known that a lossless transmission line is described by the following linear hyperbolic system

$$
\partial i(x, t) / \partial x=-C \partial u(x, t) / \partial t, \partial u(x, t) / \partial x=-L \partial i(x, t) / \partial t
$$

where $L$ and $C$ are the specific constant parameters of the line and $\Lambda$ is its length.

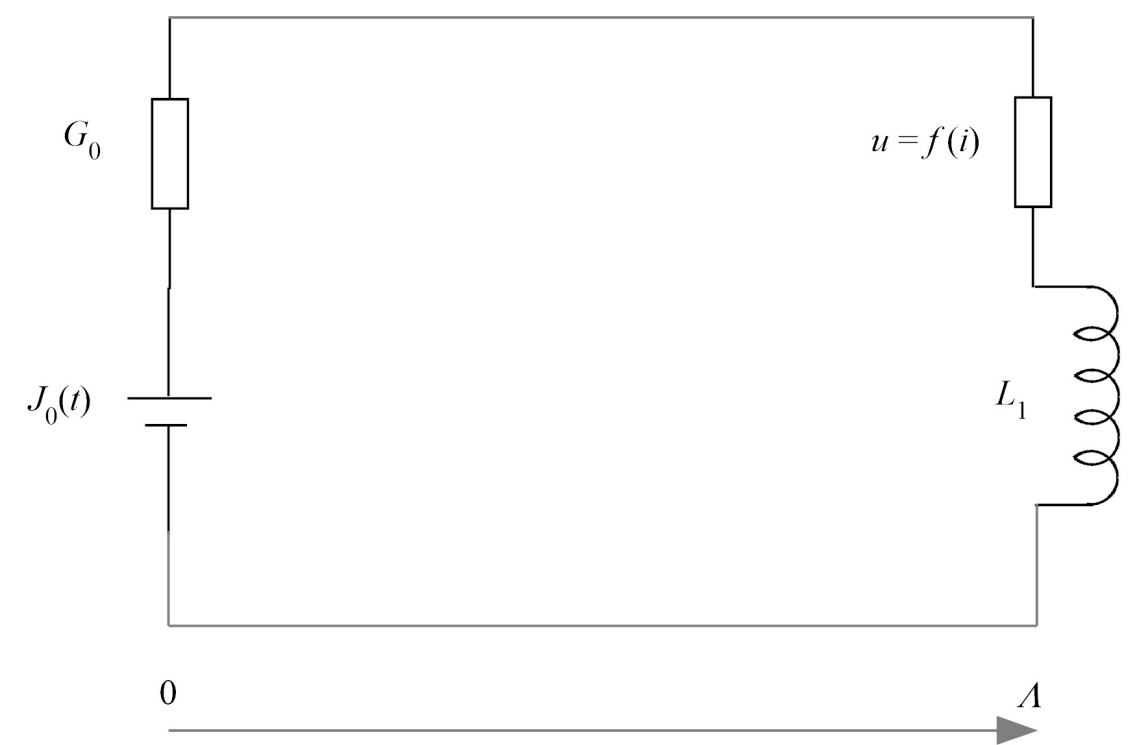

Figure 1. Lossless transmission line terminated by nonlinear load and in series connected linear inductance. 
Here we assume a polynomial nonlinearity of the resistive element $u=f(i)=\sum_{n=1}^{m} r_{n} i^{n}$. In this manner we include the example from [19].

We formulate a mixed problem for System (1): to find a solution $u(x, t), i(x, t)$ of System (1) for $(x, t) \in \Pi=\left\{(x, t) \in R^{2}:(x, t) \in[0, \Lambda] \times[0, \infty)\right\}$ with boundary conditions

$J_{0}(t)+G_{0} u(0, t)=i(0, t), t \geq 0 ; L_{1} \mathrm{~d} i(\Lambda, t) / \mathrm{d} t+\sum_{n=1}^{m} r_{n} i^{n}(\Lambda, t)=-u(\Lambda, t), t \geq 0$

and initial conditions

$$
u(x, 0)=u_{0}(x), i(x, 0)=i_{0}(x), x \in[0, \Lambda],
$$

where $u_{0}(x), i_{0}(x)$ are prescribed initial functions.

\section{Transformation of the Hyperbolic System in Diagonal Form}

The System (1) can be rewritten in matrix form $\left[\begin{array}{c}\partial u / \partial t \\ \partial i / \partial t\end{array}\right]+\left[\begin{array}{cc}0 & 1 / C \\ 1 / L & 0\end{array}\right]\left[\begin{array}{l}\partial u / \partial x \\ \partial i / \partial x\end{array}\right]=\left[\begin{array}{l}0 \\ 0\end{array}\right]$

Introducing denotations $U=\left[\begin{array}{l}u \\ i\end{array}\right], \quad A=\left[\begin{array}{cc}0 & 1 / C \\ 1 / L & 0\end{array}\right], \quad \overline{0}=\left[\begin{array}{l}0 \\ 0\end{array}\right]$ we obtain the equation

$$
\frac{\partial U}{\partial t}+A \frac{\partial U}{\partial x}=\overline{0}
$$

To transform the matrix $A=\left[\begin{array}{cc}0 & 1 / C \\ 1 / L & 0\end{array}\right]$ in diagonal form we solve the characteristic equation $\left|\begin{array}{cc}-\lambda & 1 / C \\ 1 / L & -\lambda\end{array}\right|=0$. Its roots are $\lambda_{1}=1 / \sqrt{L C}, \lambda_{2}=-1 / \sqrt{L C}$. The corresponding eigenvectors are $\left(\xi_{1}^{(1)}, \xi_{2}^{(1)}\right)=(\sqrt{C}, \sqrt{L})$, $\left(\xi_{1}^{(2)}, \xi_{2}^{(2)}\right)=(-\sqrt{C}, \sqrt{L})$ and we form the matrix $H=\left[\begin{array}{cc}\sqrt{C} & \sqrt{L} \\ -\sqrt{C} & \sqrt{L}\end{array}\right]$. Its inverse is $H^{-1}=\left[\begin{array}{cc}1 /(2 \sqrt{C}) & -1 /(2 \sqrt{C}) \\ 1 /(2 \sqrt{L}) & 1 /(2 \sqrt{L})\end{array}\right]$. Then $H A H^{-1}=\left[\begin{array}{cc}1 / \sqrt{L C} & 0 \\ 0 & -1 / \sqrt{L C}\end{array}\right]$. Introduce new variables $Z=\left[\begin{array}{c}V(x, t) \\ I(x, t)\end{array}\right]$, where $Z=H U$ and $U=H^{-1} Z$, that is,

$$
\mid \begin{array}{l|l}
V(x, t)=\sqrt{C}(x, t)+\sqrt{L} i(x, t) \\
I(x, t)=-\sqrt{C}(x, t)+\sqrt{L} i(x, t)
\end{array} \text { and } \mid \begin{aligned}
& u(x, t)=[V(x, t)-I(x, t)] /(2 \sqrt{C}) \\
& i(x, t)=[V(x, t)+I(x, t)] /(2 \sqrt{L})
\end{aligned} .
$$

Substituting $U=H^{-1} Z$ in Equation (3) we obtain $H^{-1} \partial Z / \partial t+A\left(H^{-1} \partial Z / \partial x\right)=0$ and after multiplication from the left by $H$ we obtain $\partial Z / \partial t+\left(H A H^{-1}\right) \partial Z / \partial x=0$ or 


$$
\partial V(x, t) / \partial t+[\partial V(x, t) / \partial x] / \sqrt{L C}=0, \partial I(x, t) / \partial t-[\partial I(x, t) / \partial x] / \sqrt{L C}=0 .
$$

Recall denotations $Z_{0}=\sqrt{L / C}$-characteristic impedance and $v=1 / \sqrt{L C}$-speed of propagation.

\section{Reducing the Mixed Problem to an Initial Value Problem on the Boundary}

Prior to formulate an operator corresponding to the mixed problem we consider the Cauchy problems for the characteristics of the hyperbolic system (1) (cf. [17]):

$$
\mathrm{d} \xi / \mathrm{d} \tau=1 / \sqrt{L C}, \xi(t)=x \text { and } \mathrm{d} \xi / \mathrm{d} \tau=-1 / \sqrt{L C}, \xi(t)=x
$$

for each $(x, t) \in \Pi$. Here the characteristics are constants $\lambda_{V}=1 / \sqrt{L C}>0$ and $\lambda_{I}=-1 / \sqrt{L C}<0$, and therefore continuous ones.

Denote by $T=\Lambda / v=\Lambda \sqrt{L C}$. To obtain boundary conditions with respect to the new variables we substitute

$$
\begin{gathered}
u(0, t)=(V(0, t)-I(0, t)) /(2 \sqrt{C}), \quad i(0, t)=(V(0, t)+I(0, t)) /(2 \sqrt{L}), \\
u(\Lambda, t)=(V(\Lambda, t)-I(\Lambda, t)) /(2 \sqrt{C}), \quad i(\Lambda, t)=(V(\Lambda, t)+I(\Lambda, t)) /(2 \sqrt{L})
\end{gathered}
$$

into boundary conditions (2) and get

$$
\begin{aligned}
& J_{0}(t)+(V(0, t)-I(0, t))\left(G_{0} / 2 \sqrt{C}\right)=(V(0, t)+I(0, t)) /(2 \sqrt{L}), t \geq 0 \\
& \left(L_{1} /(2 \sqrt{L})\right)[\mathrm{d}(V(\Lambda, t)+I(\Lambda, t))] / \mathrm{d} t+\sum_{n=1}^{m} r_{n}([V(\Lambda, t)+I(\Lambda, t)] /(2 \sqrt{L}))^{n} \\
& =-(V(\Lambda, t)-I(\Lambda, t)) /(2 \sqrt{C}), t \geq 0 .
\end{aligned}
$$

To obtain new initial conditions we proceed from

$$
\begin{aligned}
& V(x, t)=\sqrt{C} u(x, t)+\sqrt{L} i(x, t), \\
& I(x, t)=-\sqrt{C} u(x, t)+\sqrt{L} i(x, t)
\end{aligned} .
$$

Then

$$
\begin{aligned}
& V(x, 0)=\sqrt{C} u(x, 0)+\sqrt{L} i(x, 0)=\sqrt{C} u_{0}(x)+\sqrt{L} i_{0}(x) \equiv V_{0}(x) \\
& \begin{aligned}
I(x, 0) & =-\sqrt{C} u(x, 0)+\sqrt{L} i(x, 0) \equiv-\sqrt{C} u_{0}(x)+\sqrt{L} i_{0}(x) \\
& \equiv I_{0}(x), x \in[0, \Lambda] .
\end{aligned}
\end{aligned}
$$

Now we able to formulate a mixed problem with respect to the new variables: to find a solution of the system

$$
\begin{aligned}
& \partial V(x, t) / \partial t+1 /(\sqrt{L C}) \partial V(x, t) / \partial x=0 \\
& \partial I(x, t) / \partial t-1 /(\sqrt{L C}) \partial I(x, t) / \partial x=0
\end{aligned} \quad x \in[0, \Lambda] ; t \in[T, \infty)
$$

satisfying initial conditions

$$
V(x, 0)=V_{0}(x), I(x, 0)=I_{0}(x), x \in[0, \Lambda]
$$

and boundary conditions 


$$
\begin{aligned}
& J_{0}(t)+(V(0, t)-I(0, t))\left(G_{0} / 2 \sqrt{C}\right)=(V(0, t)+I(0, t)) /(2 \sqrt{L}), \quad t \geq T \\
& \left(L_{1} /(2 \sqrt{L})\right)[\mathrm{d}(V(\Lambda, t)+I(\Lambda, t))] / \mathrm{d} t+\sum_{n=1}^{m} r_{n}([V(\Lambda, t)+I(\Lambda, t)] /(2 \sqrt{L}))^{n} \\
& =-(V(\Lambda, t)-I(\Lambda, t)) /(2 \sqrt{C}), t \geq T .
\end{aligned}
$$

Repeating reasoning from [17] we integrate System (5) along the characteristics and obtain

$$
V(0, t-T)=V(\Lambda, t), I(0, t)=I(\Lambda, t-T)
$$

Assuming that $V(0, t)=V(t), I(\Lambda, t)=I(t)$ are unknown functions we obtain the following initial value problem equivalent to the mixed problem (5) (cf. [17]), (using denotation $\mathrm{d} V(t-T) / \mathrm{d} t=\dot{V}(t-T))$ :

$$
\begin{aligned}
V(t) & =\left(-2 \sqrt{L} J_{0}(t)+\left(G_{0} Z_{0}+1\right) V(t-T)\right) /\left(G_{0} Z_{0}-1\right) \equiv F_{V}(V, I)(t), t \geq T \\
\dot{I}(t) & =-\dot{V}(t-T)-\left[Z_{0}(V(t)-I(t-T))+\sum_{n=1}^{m} r_{n}(V(t)+I(t-T))^{n} /(2 \sqrt{L})^{n-1}\right] / L_{1} \\
& \equiv F_{I}(V, I)(t), t \geq T \\
V(t) & =V_{0}(t), I(t)=I_{0}(t), t \in[0, T] .
\end{aligned}
$$

The initial functions $V_{0}(t), I_{0}(t)$ are obtained from the initial functions

$$
u(x, 0)=u_{0}(x), i(x, 0)=i_{0}(x), x \in[0, \Lambda]
$$

after transition along the characteristics of the hyperbolic system (cf. [17]).

\section{Existence-Uniqueness of an Oscillatory Continuous Solution}

Now we are able to formulate the main problem: to find an oscillatory solution of System (6) with advanced prescribed zeroes on an interval $[T, \infty)$, where $V_{0}(t), I_{0}(t)$ are continuously differentiable prescribed oscillating functions on the initial interval $[0, T]$.

Let $S_{T}=\left\{\tau_{k}\right\}_{k=1}^{n}, n \in N$ be the prescribed set of zeroes of the initial function, that is, $V_{0}\left(\tau_{k}\right)=0, I_{0}\left(\tau_{k}\right)=0$ such that $\tau_{0}=0, \tau_{n}=T$. Besides $\max \left\{\tau_{k+1}-\tau_{k}: k=0,1, \cdots, n\right\} \leq T_{0}<\infty$.

Let $S=\left\{t_{k}\right\}_{k=0}^{\infty}$ be a strictly increasing sequence of real numbers satisfying the following conditions $(\mathrm{C})$ :

(C1) $t_{0}=T$; $\lim _{k \rightarrow \infty} t_{k}=\infty$; (C2) for every $k$ there is $s<k$ such that $t_{k}-T=t_{s}$, where $t_{s} \in S_{T} \cup S$.

It follows

$$
0 \leq \Delta=\inf \left\{t_{k+1}-t_{k}: k=0,1,2, \cdots\right\} \leq \sup \left\{t_{k+1}-t_{k}: k=0,1,2, \cdots\right\}=T_{0}<\infty .
$$

Consider the sets

$$
\begin{gathered}
M_{V}=\left\{V(.) \in C[T, \infty): V\left(t_{k}\right)=0 \text { and }|V(t)| \leq V_{0} \mathrm{e}^{\mu\left(t-t_{k}\right)}, t \in\left[t_{k}, t_{k=1}\right]\right\}, \\
M_{I}=\left\{I(.) \in C[T, \infty): I\left(t_{k}\right)=0 \text { and }|I(t)| \leq I_{0} \mathrm{e}^{\mu\left(t-t_{k}\right)}, t \in\left[t_{k}, t_{k=1}\right]\right\}
\end{gathered}
$$

$(k=0,1,2, \cdots)$, where $V_{0}, I_{0}, \mu$ are positive constants. 
Introduce the following family of pseudo-metrics

$$
\begin{aligned}
& \rho^{(k)}(V, \bar{V})=\max \left\{\mathrm{e}^{-\mu\left(t-t_{k}\right)}|V(t)-\bar{V}(t)|: t \in\left[t_{k}, t_{k+1}\right]\right\}, \\
& \rho^{(k)}(I, \bar{I})=\max \left\{\mathrm{e}^{-\mu\left(t-t_{k}\right)}|I(t)-\bar{I}(t)|: t \in\left[t_{k}, t_{k+1}\right]\right\} .
\end{aligned}
$$

The set $M_{V} \times M_{I}$ turns out into a complete uniform space with respect to the saturated family of pseudo-metrics $\rho^{(k)}((V, I),(\bar{V}, \bar{I}))=\max \left\{\rho^{(k)}(V, \bar{V}), \rho^{(k)}(I, \bar{I})\right\},(k=0,1,2, \cdots)$.

Using System (6) we define an operator

$$
B=\left(B_{V}(V, I), B_{I}(V, I)\right): M_{V} \times M_{I} \rightarrow M_{V} \times M_{I}
$$

by the formulas

$$
\begin{gathered}
B_{V}(V, I)(t):=\left(-2 \sqrt{L} J_{0}(t)+\left(G_{0} Z_{0}+1\right) V(t-T)\right) /\left(G_{0} Z_{0}-1\right) \\
\equiv F_{V}(V, I)(t), t \in\left[t_{k}, t_{k+1}\right],(k=0,1,2, \cdots) \\
B_{I}(V, I)(t):=\int_{t_{k}}^{t} F_{I}(V, I)(s) \mathrm{d} s-\left(\left(t-t_{k}\right) /\left(t_{k+1}-t_{k}\right)\right) \int_{t_{k}}^{t_{k+1}} F_{I}(V, I)(s) \mathrm{d} s, \\
t \in\left[t_{k}, t_{k+1}\right],(k=0,1,2, \cdots)
\end{gathered}
$$

Remark 1.

1) In the above definitions of the operator $B$ the functions $V(t-T)$ and $I(t-T)$ in the right-hand side are substituted by the initial functions in the interval $[T, 2 T]$.

2) The conformity condition (CC)

$$
\dot{I}(T)=-\dot{V}(0)-\left(Z_{0}[V(T)-I(0)]+\sum_{n=1}^{m} r_{n}[V(T)+I(0)]^{n} /(2 \sqrt{L})^{n-1}\right) / L_{1}
$$

becomes $\dot{I}(T)=-\dot{V}(0)$. It is necessary when we look for a smooth solution.

We call a solution of System (6) the solution of the operator equation $(V, I)=\left(B_{V}(V, I), B_{I}(V, I)\right)$.

The following lemma is valid:

Lemma 1. Let be the source function satisfies $J_{0}\left(\tau_{k}\right)=0, J_{0}\left(t_{k}\right)=0,\left|J_{0}(t)\right| \leq I_{0} \mathrm{e}^{\mu\left(t-t_{k}\right)}, t \in\left[t_{k}, t_{k+1}\right]$. Problem (6) has a solution $(V(),. I().) \in M_{V} \times M_{I}$ iff the operator $B$ has a fixed point in $M_{V} \times M_{I}$, that is,

$$
(V, I)=\left(B_{V}(V, I), B_{I}(V, I)\right) \text {. }
$$

Proof. Let $(V(),. I().) \in M_{V} \times M_{I}$ be a solution of System (6). We show that $V=B_{V}\left((V, I) ; I=B_{I}(V, I)\right.$.

For the first equation an existence of solution is obviously equivalent to $V=B_{V}(V, I)$.

To prove the same for the second equation we integrate the second equation on the interval

$$
\begin{aligned}
& {\left[t_{k}, t\right] \subset\left[t_{k}, t_{k+1}\right](k=0,1,2, \cdots) \text { and get }} \\
& I(t)=\int_{t_{k}}^{t} F_{I}(V, I)(s) \mathrm{d} s \Rightarrow 0=I\left(t_{k+1}\right)=\int_{t_{k}}^{t_{k+1}} F_{I}(V, I)(s) \mathrm{d} s \Rightarrow \int_{t_{k}}^{t_{k+1}} F_{I}(V, I)(s) \mathrm{d} s=0
\end{aligned}
$$


Therefore $I(t)$ satisfies

$$
\begin{aligned}
I(t) & =\int_{t_{k}}^{t} F_{I}(V, I)(s) \mathrm{d} s-\left(\left(t-t_{k}\right) /\left(t_{k+1}-t_{k}\right)\right) \int_{t_{k}}^{t_{k+1}} F_{I}(V, I)(s) \mathrm{d} s, t \in\left[t_{k}, t_{k+1}\right] . \\
& \Leftrightarrow I=B_{I}(V, I) .
\end{aligned}
$$

Therefore $(V(),. I()$.$) is a fixed point of the operator B$.

Conversely, let $(V(),. I().) \in M_{V} \times M_{I}$ be a fixed point of the operator $B$, that is $V=B_{V}(V, I) ; I=B_{I}(V, I)$. We prove the implication for the second equation. Indeed, we have

$$
\begin{aligned}
& I(t)=\int_{t_{k}}^{t} F_{I}(V, I)(s) \mathrm{d} s-\left(\left(t-t_{k}\right) /\left(t_{k+1}-t_{k}\right)\right) \int_{t_{k}}^{t_{k+1}} F_{I}(V, I)(s) \mathrm{d}, t \in\left[t_{k}, t_{k+1}\right],(k=0,1,2, \cdots) \\
& \text { Since }\left|\left(t-t_{k}\right) /\left(t_{k+1}-t_{k}\right)\right| \leq 1, t \in\left[t_{k}, t_{k+1}\right] \text { we estimate the second term: } \\
& \left|\int_{t_{k}}^{t_{k+1}} F_{I}(V, I)(s) \mathrm{d}\right| \mid \\
& \leq\left|\int_{t_{k}}^{t_{k+1}} \dot{V}(s-T) \mathrm{d} s\right|+\left[Z_{0}\left(\int_{t_{k}}^{t_{k+1}}|V(s)| \mathrm{d} s+\int_{t_{k}}^{t_{k+1}}|I(s-T)| \mathrm{d} s\right)+\sum_{n=1}^{m}\left|r_{n}\right| /(2 \sqrt{L})^{n-1} \int_{t_{k}}^{t_{k+1}}(|V(s)|+|I(s-T)|)^{n} \mathrm{~d} s\right] / L_{1} \\
& \leq\left|V\left(t_{k+1}-T\right)-V\left(t_{k}-T\right)\right|+\left(Z_{0} V_{0} / L_{1}\right)^{t_{k+1}} \int_{t_{k}}^{\mu\left(s-t_{k}\right)} \mathrm{d} s+\left(Z_{0} V_{0} / L_{1}\right) \int_{t_{k}}^{t_{k+1}} \mathrm{e}^{\mu\left(s-T-t_{k}\right)} \mathrm{d} s \\
& +\sum_{n=1}^{m}\left(\left|r_{n}\right| / L_{1}(2 \sqrt{L})^{n-1}\right)^{t_{k+1}} \int_{t_{k}}\left(V_{0} \mathrm{e}^{\mu\left(s-t_{k}\right)}+I_{0} \mathrm{e}^{\mu\left(s-T-t_{k}\right)}\right)^{n} \mathrm{~d} s \\
& \leq\left(Z_{0} / \mu L_{1}\right)\left[V_{0}\left(\mathrm{e}^{\mu\left(t_{k+1}-t_{k}\right)}-1\right)+I_{0} \mathrm{e}^{-\mu T}\left(\mathrm{e}^{\mu\left(t_{k+1}-t_{k}\right)}-1\right)\right]+\left(1 / L_{1}\right) \sum_{n=1}^{m}\left(\left|r_{n}\right| /(2 \sqrt{L})^{n-1}\right)\left(V_{0}+I_{0} \mathrm{e}^{-\mu T}\right)^{n} \int_{t_{k}}^{t_{k+1}} \mathrm{e}^{n \mu\left(s-t_{k}\right)} \mathrm{d} s \\
& \leq\left(Z_{0} / \mu L_{1}\right)\left(\mathrm{e}^{\mu T_{0}}-1\right)\left(V_{0}+I_{0} \mathrm{e}^{-\mu T}\right)+\left(1 / L_{1}\right) \sum_{n=1}^{m}\left(\left|r_{n}\right| /(2 \sqrt{L})^{n-1}\right)\left(V_{0}+I_{0} \mathrm{e}^{-\mu T}\right)^{n}\left[\left(\mathrm{e}^{n \mu T_{0}}-1\right) /(n \mu)\right] \\
& \leq\left(\mathrm{e}^{\mu T_{0}}-1\right)\left(V_{0}+I_{0} \mathrm{e}^{-\mu T}\right) /\left(\mu L_{1}\right)\left(Z_{0}+\sum_{n=1}^{m}\left|r_{n}\right|\left(\left(V_{0}+I_{0} \mathrm{e}^{-\mu T}\right) /(2 \sqrt{L})\right)^{n-1}\left(\mathrm{e}^{(n-1) \mu T_{0}}+\cdots+1\right) / n\right) \equiv M(\mu)
\end{aligned}
$$$$
\text { Let us assume that }\left|\int_{t_{k}}^{t_{k+1}} F_{I}(V, I)(s) \mathrm{d} s\right|=\gamma>0 \text {. But we have obtained that }
$$$$
\gamma \leq M(\mu) \text {. For sufficiently large } \mu>0 \text { (and sufficiently small } T_{0}>0 \text { ) }
$$$$
\mu T_{0}=\text { const we have } M(\mu)<\gamma \text {. The obtained contradiction implies }
$$$$
\int_{t_{k}}^{t_{k+1}} F_{I}(V, I)(s) \mathrm{d} s=0 \text {. It follows } I(t)=\int_{t_{k}}^{t} F_{I}(V, I)(s) \mathrm{d} s \text { and after a differentia- }
$$
tion we obtain (6).

Lemma 1 is thus proved.

Theorem 1. Let the following conditions be fulfilled:

1) The initial functions $V_{0}(),. I_{0}(.) \in C[0, T]$ satisfy conditions

$$
\begin{aligned}
& V_{0}\left(\tau_{k}\right)=0 \text { and }\left|V_{0}(t)\right| \leq V_{0} \mathrm{e}^{\mu\left(t-\tau_{k}\right)},\left|I_{0}(t)\right| \leq I_{0} \mathrm{e}^{\mu\left(t-\tau_{k}\right)}, t \in\left[\tau_{k}, \tau_{k+1}\right] \text {; } \\
& \text { 2) } J_{0}\left(\tau_{k}\right)=0, J_{0}\left(t_{k}\right)=0,\left|J_{0}(t)\right| \leq I_{0} \mathrm{e}^{\mu\left(t-t_{k}\right)}, t \in\left[t_{k}, t_{k+1}\right] ; \\
& \text { 3) }\left(2 I_{0} \sqrt{L}+\left|G_{0} Z_{0}-1\right| V_{0} \mathrm{e}^{-\mu T}\right) /\left(G_{0} Z_{0}+1\right) \leq V_{0} ;
\end{aligned}
$$


4) $V_{0} \mathrm{e}^{-\mu T}+\left(\mathrm{e}^{\mu T_{0}}\left(V_{0}+I_{0} \mathrm{e}^{-\mu T}\right) /\left(\mu L_{1}\right)\right)\left(Z_{0}+\sum_{n=1}^{m}\left(\left|r_{n}\right| / n(2 \sqrt{L})^{n-1}\right)\left(V_{0}+I_{0} \mathrm{e}^{-\mu T}\right)^{n-1}\left(\mathrm{e}^{(n-1) \mu T_{0}}+\cdots+1\right)\right) \leq I_{0}$;

5) $K_{V}=\left|G_{0} Z_{0}-1\right| \mathrm{e}^{-\mu T} /\left(G_{0} Z_{0}+1\right)<1$;

6) $K_{I}=\mathrm{e}^{-\mu T}+\left(\mathrm{e}^{\mu T_{0}}\left(1+\mathrm{e}^{-\mu T}\right) /\left(\mu L_{1}\right)\right)\left(Z_{0}+\sum_{n=1}^{m}\left|r_{n}\right|\left(\mathrm{e}^{(n-1) \mu T_{0}}+\cdots+1\right)\left(\left(V_{0}+I_{0} \mathrm{e}^{-\mu T}\right) /(2 \sqrt{L})\right)^{n-1}\right)<1$.

Then there exists a unique oscillatory solution of System (6), belonging to $M_{V} \times M_{I}$.

Proof. We show that the operator $B=\left(B_{V}, B_{I}\right)$ maps $M_{V} \times M_{I}$ into itself. Indeed, $\left(B_{V}(t), B_{I}(t)\right)$ is continuous on $[0, \infty)$. It is easy to check that (recall that $\tau_{n}=T$ )

$$
\begin{gathered}
B_{V}(V, I)\left(\tau_{n}\right)=\left(2 \sqrt{L} J_{0}\left(\tau_{n}\right)+\left(G_{0} Z_{0}-1\right) V\left(\tau_{n}-T\right)\right) /\left(G_{0} Z_{0}\right)+1=0 ; \\
B_{V}(V, I)\left(t_{k}\right)=\left(2 \sqrt{L} J_{0}\left(t_{k}\right)+\left(G_{0} Z_{0}-1\right) V\left(t_{k}-T\right)\right) /\left(G_{0} Z_{0}\right)+1=0 ; \\
B_{I}(V, I)\left(\tau_{n}\right)=\int_{\tau_{n}}^{\tau_{n}} F_{I}(V, I)(s) \mathrm{d} s-\left(\left(\tau_{n}-\tau_{n}\right) /\left(t_{1}-\tau_{n}\right)\right) \int_{\tau_{n}}^{t_{1}} F_{I}(V, I)(s) \mathrm{d} s=0 ; \\
B_{I}(V, I)\left(t_{k}\right):=\int_{t_{k}}^{t_{k}} F_{I}(V, I)(s) \mathrm{d} s-\left(\left(t_{k}-t_{k}\right) /\left(t_{k+1}-t_{k}\right)\right) \int_{t_{k}}^{t_{k+1}} F_{I}(V, I)(s) \mathrm{d} s=0,
\end{gathered}
$$

We show that $\left|B_{V}(V, I)(t)\right| \leq V_{0} \mathrm{e}^{\mu\left(t-t_{k}\right)}$ and $\left|B_{I}(V, I)(t)\right| \leq I_{0} \mathrm{e}^{\mu\left(t-t_{k}\right)}$ for $t \in\left[t_{k}, t_{k+1}\right]$.

First we notice that $\left|\left(t-t_{k}\right) /\left(t_{k+1}-t_{k}\right)\right| \leq 1, t \in\left[t_{k}, t_{k+1}\right]$.

For sufficiently large $\mu$ and $t \in\left[t_{k}, t_{k+1}\right]$ for the first component we obtain

$$
\begin{aligned}
\left|B_{V}(V, I)(t)\right| & \leq\left(2 \sqrt{L}\left|J_{0}(t)\right|+\left|G_{0} Z_{0}-1\right||V(t-T)|\right) /\left(G_{0} Z_{0}+1\right) \\
& \leq \mathrm{e}^{\mu\left(t-t_{k}\right)}\left(2 I_{0} \sqrt{L}+\left|G_{0} Z_{0}-1\right| V_{0} \mathrm{e}^{-\mu T}\right) /\left(G_{0} Z_{0}+1\right) \leq V_{0} \mathrm{e}^{\mu\left(t-t_{k}\right)} .
\end{aligned}
$$

For the second one we have

$$
\begin{aligned}
& \left|B_{I}(V, I)(s)(t)\right| \leq\left|\int_{t_{k}}^{t} F_{I}(V, I)(s)(s) \mathrm{d} s\right|+\left|\left(t-t_{k}\right) /\left(t_{k+1}-t_{k}\right)\right| \int_{t_{k}}^{t_{k+1}} F_{I}(V, I)(s)(s) \mathrm{d} s \mid=B_{1}+B_{2} ; \\
& B_{1}=\left|\int_{t_{k}}^{t} F_{I}(V, I)(s) \mathrm{d} s\right| \\
& \leq\left|\int_{t_{k}}^{t} \dot{V}(s-T) \mathrm{d} s\right|+\frac{Z_{0}}{L_{1}}\left(\int_{t_{k}}^{t}|V(s)| \mathrm{d} s+\int_{t_{k}}^{t}|I(s-T)| \mathrm{d} s\right)+\sum_{n=1}^{m} \frac{\left|r_{n}\right|}{L_{1}(2 \sqrt{L})^{n-1}} \int_{t_{k}}^{t}(|V(s)|+|I(s-T)|)^{n} \mathrm{~d} s \\
& \leq V_{0} \mathrm{e}^{-\mu T}+\left(Z_{0} / L_{1}\right)\left(V_{0} \int_{t_{k}}^{t} \mathrm{e}^{\mu\left(s-t_{k}\right)} \mathrm{d} s+I_{0} \int_{t_{k}}^{t} \mathrm{e}^{\mu\left(s-T-t_{k}\right)} \mathrm{d} s\right)+\sum_{n=1}^{m}\left(\left|r_{n}\right| / L_{1}(2 \sqrt{L})^{n-1}\right) \int_{t_{k}}^{t}\left(V_{0} \mathrm{e}^{\mu\left(s-t_{k}\right)}+I_{0} \mathrm{e}^{\mu\left(s-T-t_{k}\right)}\right)^{n} \mathrm{~d} s \\
& \leq V_{0} \mathrm{e}^{-\mu T}+\left(Z_{0} / \mu L_{1}\right)\left(\mathrm{e}^{\mu\left(t-t_{k}\right)}-1\right)\left(V_{0}+I_{0} \mathrm{e}^{-\mu T}\right)+\sum_{n=1}^{m}\left(\left|r_{n}\right| / L_{1}(2 \sqrt{L})^{n-1}\right)\left(V_{0}+I_{0} \mathrm{e}^{-\mu T}\right)^{n} \int_{t_{k}}^{t} \mathrm{e}^{n \mu\left(s-t_{k}\right)} \mathrm{d} s \\
& \leq V_{0} \mathrm{e}^{-\mu T}+Z_{0}\left(\mathrm{e}^{\mu\left(t-t_{k}\right)}-1\right)\left(V_{0}+I_{0} \mathrm{e}^{-\mu T}\right) /\left(\mu L_{1}\right)+\sum_{n=1}^{m}\left(\left|r_{n}\right| / L_{1} n \mu(2 \sqrt{L})^{n-1}\right)\left(V_{0}+I_{0} \mathrm{e}^{-\mu T}\right)^{n}\left(\mathrm{e}^{n \mu\left(t-t_{k}\right)}-1\right) \\
& \leq V_{0} \mathrm{e}^{-\mu T}+\left(\mathrm{e}^{\mu\left(t-t_{k}\right)}\left(V_{0}+I_{0} \mathrm{e}^{-\mu T}\right) /\left(\mu L_{1}\right)\right)\left(Z_{0}+\sum_{n=1}^{m}\left(\left|r_{n}\right| / n(2 \sqrt{L})^{n-1}\right)\left(V_{0}+I_{0} \mathrm{e}^{-\mu T}\right)^{n-1}\left(\mathrm{e}^{(n-1) \mu T_{0}}+\cdots+1\right)\right) .
\end{aligned}
$$


From Lemma 1 we have

$$
\begin{aligned}
& B_{2} \leq\left|\left(t-t_{k}\right) /\left(t_{k+1}-t_{k}\right)\right| \int_{t_{k}}^{t_{k+1}} F_{I}(V, I)(s) \mathrm{d} s|\leq| \int_{t_{k}}^{t_{k+1}} F_{I}(V, I)(s) \mathrm{d} s \mid \\
& \leq\left(\left(\mathrm{e}^{\mu T_{0}}-1\right)\left(V_{0}+I_{0} \mathrm{e}^{-\mu T}\right) /\left(\mu L_{1}\right)\right)\left(Z_{0}+\sum_{n=1}^{m}\left(\left|r_{n}\right| / n(2 \sqrt{L})^{n-1}\right)\left(V_{0}+I_{0} \mathrm{e}^{-\mu T}\right)^{n-1}\left(\mathrm{e}^{(n-1) \mu T_{0}}+\cdots+1\right)\right) \\
& \text { Therefore } \\
& \left|B_{I}(V, I)(s)(t)\right| \quad \mathrm{e}^{-\mu T}+\mathrm{e}^{\mu\left(t-t_{k}\right)}\left(\left(V_{0}+I_{0} \mathrm{e}^{-\mu T}\right) /\left(\mu L_{1}\right)\right)\left(Z_{0}+\sum_{n=1}^{m}\left(\left|r_{n}\right| / n(2 \sqrt{L})^{n-1}\right)\left(V_{0}+I_{0} \mathrm{e}^{-\mu T}\right)^{n-1}\left(\mathrm{e}^{(n-1) \mu T_{0}}+\cdots+1\right)\right) \\
& +\left(\left(\mathrm{e}^{\mu T_{0}}-1\right)\left(V_{0}+I_{0} \mathrm{e}^{-\mu T}\right) /\left(\mu L_{1}\right)\right)\left(Z_{0}+\sum_{n=1}^{m}\left(\left|r_{n}\right| / n(2 \sqrt{L})^{n-1}\right)\left(V_{0}+I_{0} \mathrm{e}^{-\mu T}\right)^{n-1}\left(\mathrm{e}^{(n-1) \mu T_{0}}+\cdots+1\right)\right) \\
& \leq \mathrm{e}^{\mu\left(t-t_{k}\right)}\left[V_{0} \mathrm{e}^{-\mu T}+\left(\mathrm{e}^{\mu T_{0}}\left(V_{0}+I_{0} \mathrm{e}^{-\mu T}\right) /\left(\mu L_{1}\right)\right)\left(Z_{0}+\sum_{n=1}^{m}\left(\left|r_{n}\right| / n(2 \sqrt{L})^{n-1}\right)\left(V_{0}+I_{0} \mathrm{e}^{-\mu T}\right)^{n-1}\left(\mathrm{e}^{(n-1) \mu T_{0}}+\cdots+1\right)\right)\right] \\
& \leq \mathrm{e}^{\mu\left(t-t_{k}\right)} I_{0} .
\end{aligned}
$$

It remains to show that the operator $B$ is contractive one.

For the first component we obtain

$$
\begin{aligned}
& \left|B_{V}(V, I)(t)-B_{V}(\bar{V}, \bar{I})(t)(t)\right| \\
& \leq\left|G_{0} Z_{0}-1\right||V(t-T)-\bar{V}(t-T)| /\left(G_{0} Z_{0}+1\right) \\
& \leq \mathrm{e}^{\mu\left(t-t_{k}\right)}\left|G_{0} Z_{0}-1\right| \rho^{(k)}(V, \bar{V}) \mathrm{e}^{-\mu T} /\left(G_{0} Z_{0}+1\right) \\
& \leq \mathrm{e}^{\mu\left(t-t_{k}\right)}\left|G_{0} Z_{0}-1\right| \mathrm{e}^{-\mu T} \rho^{(k)}((V, I),(\bar{V}, \bar{I})) /\left(G_{0} Z_{0}+1\right)
\end{aligned}
$$

It follows

$$
\begin{aligned}
& \rho^{(k)}\left(B_{V}(V, I), B_{V}(\bar{V}, \bar{I})\right) \\
& \leq\left|G_{0} Z_{0}-1\right| \mathrm{e}^{-\mu T} \rho^{(k)}((V, I),(\bar{V}, \bar{I})) /\left(G_{0} Z_{0}+1\right) \\
& \equiv K_{I} \rho^{(k)}((V, I),(\bar{V}, \bar{I}))
\end{aligned}
$$

For the second one in view of the estimate from Lemma 1 we have

$$
\begin{aligned}
& \left|B_{I}(V, I)(t)-B_{I}(\bar{V}, \bar{I})(t)\right| \\
& \leq\left|\int_{t_{k}}^{t} F_{I}(V, I)(s) \mathrm{d} s-\int_{t_{k}}^{t} F_{I}(\bar{V}, \bar{I})(s) \mathrm{d} s\right| \\
& +\left|\left(t-t_{k}\right) /\left(t_{k+1}-t_{k}\right)\right|\left|\int_{t_{k}}^{t_{k+1}} F_{I}(V, I)(s) \mathrm{d} s-\int_{t_{k}}^{t_{k+1}} F_{I}(\bar{V}, \bar{I})(s) \mathrm{d} s\right| \\
& \equiv B_{1}+B_{2} \\
& B_{1}=\left|\int_{t_{k}}^{t} F_{I}(V, I)(s) \mathrm{d} s-\int_{t_{k}}^{t} F_{I}(\bar{V}, \bar{I})(s) \mathrm{d} s\right| \\
& \leq\left|\int_{t_{k}}^{t}(\dot{V}(s-T)-\dot{\bar{V}}(s-T)) \mathrm{d} s\right|+\left(Z_{0} / L_{1}\right)\left(\int_{t_{k \leq}}^{t}|V(s)-\bar{V}(s)| \mathrm{d} s+\int_{t_{k}}^{t}|I(s-T)-\bar{I}(s-T)| \mathrm{d} s\right)
\end{aligned}
$$




$$
\begin{aligned}
& +\sum_{n=1}^{m}\left|r_{n}\right| / L_{1}(2 \sqrt{L})^{n-1} \int_{t_{k}}^{t} n\left(V_{0} \mathrm{e}^{\mu\left(s-t_{k}\right)}+I_{0} \mathrm{e}^{\mu\left(s-T-t_{k}\right)}\right)^{n-1}(|V(s)-\bar{V}(s)|+|I(s-T)-\bar{I}(s-T)|) \mathrm{d} s \\
& \leq|V(s-T)-\bar{V}(s-T)|+\left(Z_{0} / L_{1}\right)\left(\rho^{(k)}(V, \bar{V}) \int_{t_{k}}^{t} \mathrm{e}^{\mu\left(s-t_{k}\right)} \mathrm{d} s+\rho^{(k)}(I, \bar{I}) \int_{t_{k}}^{t} \mathrm{e}^{\mu\left(s-T-t_{k}\right)} \mathrm{d} s\right) \\
& +\sum_{n=1}^{m}\left|r_{n}\right| / L_{1}(2 \sqrt{L})^{n-1} \int_{t_{k}}^{t} n\left(V_{0}+I_{0} \mathrm{e}^{-\mu T}\right)^{n-1} \mathrm{e}^{(n-1) \mu\left(s-t_{k}\right)}\left(\rho^{(k)}(V, \bar{V}) \mathrm{e}^{\mu\left(s-t_{k}\right)}+\rho^{(k)}(I, \bar{I}) \mathrm{e}^{\mu\left(s-T-t_{k}\right)}\right) \mathrm{d} s \\
& \leq \mathrm{e}^{-\mu T} \rho^{(k)}(V, \bar{V})+\left(Z_{0}\left(\mathrm{e}^{\mu\left(t-t_{k}\right)}-1\right) /\left(\mu L_{1}\right)\right)\left(\rho^{(k)}(V, \bar{V})+\rho^{(k)}(I, \bar{I}) \mathrm{e}^{-\mu T}\right) \\
& +\sum_{n=1}^{m}\left|r_{n}\right| n\left(V_{0}+I_{0} \mathrm{e}^{-\mu T}\right)^{n-1}\left(\rho^{(k)}(V, \bar{V})+\rho^{(k)}(I, \bar{I}) \mathrm{e}^{-\mu T}\right) /\left(L_{1}(2 \sqrt{L})^{n-1}\right) \int_{t_{k}}^{t} \mathrm{e}^{n \mu\left(s-t_{k}\right)} \mathrm{d} s \\
& \leq \rho^{(k)}((V, I),(\bar{V}, \bar{I}))\left[\mathrm{e}^{\mu\left(t-t_{k}\right)} \mathrm{e}^{-\mu T}+\mathrm{e}^{\mu\left(t-t_{k}\right)}\left(\left(1+\mathrm{e}^{-\mu T}\right) /\left(\mu L_{1}\right)\right)\left(Z_{0}+\sum_{n=1}^{m}\left|r_{n}\right| n\left(\left(V_{0}+I_{0} \mathrm{e}^{-\mu T}\right) /(2 \sqrt{L})\right)^{n-1}\left(\mathrm{e}^{n \mu\left(t-t_{k}\right)}-1\right) / n\right)\right] \\
& \leq \mathrm{e}^{\mu\left(t-t_{k}\right)} \rho^{(k)}((V, I),(\bar{V}, \bar{I}))\left[\mathrm{e}^{-\mu T}+\left(\left(1+\mathrm{e}^{-\mu T}\right) /\left(\mu L_{1}\right)\right)\left(Z_{0}+\sum_{n=1}^{m}\left|r_{n}\right|\left(\left(V_{0}+I_{0} \mathrm{e}^{-\mu T}\right)(2 \sqrt{L})\right)^{n-1}\left(\mathrm{e}^{(n-1) \mu T_{0}}+\cdots+1\right)\right)\right] \\
& B_{2} \leq\left|\left(t-t_{k}\right) /\left(t_{k+1}-t_{k}\right)\right|\left|\int_{t_{k}}^{t_{k+1}} F_{I}(V, I)(s) \mathrm{d} s-\int_{t_{k}}^{t_{k+1}} F_{I}(\bar{V}, \bar{I})(s) \mathrm{d} s\right| \\
& \leq\left|\int_{t_{k}}^{t_{k+1}}(\dot{V}(s-T)-\dot{\bar{V}}(s-T)) \mathrm{d} s\right|+\left(Z_{0} / L_{1}\right)\left(\int_{t_{k}}^{t_{k+1}}|V(s)-\bar{V}(s)| \mathrm{d} s+\int_{t_{k}}^{t_{k+1}}|I(s-T)-\bar{I}(s-T)| \mathrm{d} s\right) \\
& +\sum_{n=1}^{m}\left|r_{n}\right| / L_{1}(2 \sqrt{L})^{n-1} \int_{t_{k}}^{t_{k+1}} n\left(V_{0} \mathrm{e}^{\mu\left(s-t_{k}\right)}+I_{0} \mathrm{e}^{\mu\left(s-T-t_{k}\right)}\right)^{n-1}(|V(s)-\bar{V}(s)|+|I(s-T)-\bar{I}(s-T)|) \mathrm{d} s \\
& \leq\left(Z_{0} / L_{1}\right)\left(\rho^{(k)}(V, \bar{V}) \int_{t_{k}}^{t_{k+1}} \mathrm{e}^{\mu\left(s-t_{k}\right)} \mathrm{d} s+\rho^{(k)}(I, \bar{I}) \int_{t_{k}}^{t_{k+1}} \mathrm{e}^{\mu\left(s-T-t_{k}\right)} \mathrm{d} s\right) \\
& +\sum_{n=1}^{m}\left|r_{n}\right| / L_{1}(2 \sqrt{L})^{n-1} \int_{t_{k}}^{t_{k}+1} n\left(V_{0}+I_{0} \mathrm{e}^{-\mu T}\right)^{n-1} \mathrm{e}^{(n-1) \mu\left(s-t_{k}\right)}\left(\rho^{(k)}(V, \bar{V}) \mathrm{e}^{\mu\left(s-t_{k}\right)}+\rho^{(k)}(I, \bar{I}) \mathrm{e}^{\mu\left(s-T-t_{k}\right)}\right) \mathrm{d} s \\
& \leq\left(Z_{0} / \mu L_{1}\right)\left(\mathrm{e}^{\mu\left(t_{k+1}-t_{k}\right)}-1\right)\left(\rho^{(k)}(V, \bar{V})+\rho^{(k)}(I, \bar{I}) \mathrm{e}^{-\mu T}\right) \\
& +\left(\left(\rho^{(k)}(V, \bar{V})+\rho^{(k)}(I, \bar{I}) \mathrm{e}^{-\mu T}\right) / L_{1}\right) \sum_{n=1}^{m}\left|r_{n}\right| n\left(\left(V_{0}+I_{0} \mathrm{e}^{-\mu T}\right) /(2 \sqrt{L})\right) \int_{t_{k}}^{n-1} \mathrm{e}^{t_{k+1}} \mathrm{e}^{n \mu\left(s-t_{k}\right)} \mathrm{d} s \\
& \leq \rho^{(k)}((V, I),(\bar{V}, \bar{I}))\left[\left(1+\mathrm{e}^{-\mu T}\right) /\left(\mu L_{1}\right)\left(Z_{0}\left(\mathrm{e}^{\mu T_{0}}-1\right)+\sum_{n=1}^{m}\left|r_{n}\right| n\left(\left(V_{0}+I_{0} \mathrm{e}^{-\mu T}\right) /(2 \sqrt{L})\right)^{n-1}\left(\left(\mathrm{e}^{n \mu T_{0}}-1\right) / n\right)\right)\right] \\
& \leq \rho^{(k)}((V, I),(\bar{V}, \bar{I}))\left(\left(\mathrm{e}^{\mu T_{0}}-1\right)\left(1+\mathrm{e}^{-\mu T}\right) /\left(\mu L_{1}\right)\right)\left[Z_{0}+\sum_{n=1}^{m}\left|r_{n}\right|\left(\left(V_{0}+I_{0} \mathrm{e}^{-\mu T}\right) /(2 \sqrt{L})\right)^{n-1}\left(\mathrm{e}^{(n-1) \mu T_{0}}+\cdots+1\right)\right]
\end{aligned}
$$

Therefore

$$
\begin{aligned}
& \left|B_{I}(V, I)(t)-B_{I}(\bar{V}, \bar{I})(t)\right| \\
& \leq \mathrm{e}^{\mu\left(t-t_{k}\right)} \rho^{(k)}((V, I),(\bar{V}, \bar{I}))\left[\mathrm{e}^{-\mu T}+\left(\left(1+\mathrm{e}^{-\mu T}\right) /\left(\mu L_{1}\right)\right)\left(Z_{0}+\sum_{n=1}^{m}\left|r_{n}\right|\left(\left(V_{0}+I_{0} \mathrm{e}^{-\mu T}\right) /(2 \sqrt{L})\right)^{n-1}\left(\mathrm{e}^{(n-1) \mu T_{0}}+\cdots+1\right)\right)\right] \\
& +\rho^{(k)}((V, I),(\bar{V}, \bar{I}))\left(\mathrm{e}^{\mu T_{0}}-1\right)\left(\left(1+\mathrm{e}^{-\mu T}\right) /\left(\mu L_{1}\right)\right)\left[Z_{0}+\sum_{n=1}^{m}\left|r_{n}\right|\left(\left(V_{0}+I_{0} e^{-\mu T}\right) /(2 \sqrt{L})\right)^{n-1}\left(e^{(n-1) \mu T_{0}}+\cdots+1\right)\right] \\
& \leq \mathrm{e}^{\mu\left(t-t_{k}\right)}\left[\mathrm{e}^{-\mu T}+\mathrm{e}^{\mu T_{0}}\left(\left(1+\mathrm{e}^{-\mu T}\right) /\left(\mu L_{1}\right)\right)\left(Z_{0}+\sum_{n=1}^{m}\left|r_{n}\right|\left(\mathrm{e}^{(n-1) \mu T_{0}}+\cdots+1\right)\left(\left(V_{0}+I_{0} \mathrm{e}^{-\mu T}\right) /(2 \sqrt{L})\right)^{n-1}\right)\right] \rho^{(k)}((V, I),(\bar{V}, \bar{I}))
\end{aligned}
$$


It follows

$$
\rho^{(k)}\left(B_{I}(V, I), B_{I}(\bar{V}, \bar{I})\right) \leq K_{I} \rho^{(k)}((V, I),(\bar{V}, \bar{I}))
$$

where

$$
K_{I}=\mathrm{e}^{-\mu T}+\mathrm{e}^{\mu T_{0}}\left(\left(1+\mathrm{e}^{-\mu T}\right) /\left(\mu L_{1}\right)\right)\left(Z_{0}+\sum_{n=1}^{m}\left|r_{n}\right|\left(\mathrm{e}^{(n-1) \mu T_{0}}+\cdots+1\right)\left(\left(V_{0}+I_{0} \mathrm{e}^{-\mu T}\right) /(2 \sqrt{L})\right)^{n-1}\right) \text {. }
$$

Consequently

$$
\rho^{(k)}\left(\left(B_{V}(V, I), B_{I}(V, I)\right),\left(B_{V}(\bar{V}, \bar{I}), B_{I}(\bar{V}, \bar{I})\right)\right) \leq K \rho^{(k)}((V, I),(\bar{V}, \bar{I})),
$$

where $K=\max \left\{K_{V}, K_{I}\right\}<1$.

Therefore the operator $B$ is contractive one (cf. [17]) and its unique fixed point is an oscillatory solution of (6).

Theorem 1 is thus proved.

\section{Numerical Example}

Our goal here is to check the inequalities of the main Theorem 1 for the 7-th order polynomial. It is natural to assume $V_{0}=I_{0}$.

Consider a transmission line with specific parameters $L=0.2 \mu \mathrm{H} / \mathrm{m}$;

$C=9 \mathrm{pF} / \mathrm{m} ; \Lambda=10 \mathrm{~m} ; L_{1}=0.2 \mu \mathrm{H} ; G_{0}=1 / 35$. Then

$Z_{0}=\sqrt{L / C}=\sqrt{0.2 \times 10^{-6} / 9 \times 10^{-12}}=10^{3} \times 0.149=149 \Omega ;$

$T=\Lambda \sqrt{L C}=10 \sqrt{\left(0.2 \times 10^{-6}\right) \times\left(9 \times 10^{-12}\right)}=10 \times 1.34 \times 10^{-9}=1.34 \times 10^{-8}$. The $V-I$ characteristic of the nonlinear element is $u=R_{s 0}\left(i+\alpha i^{7}\right)=R_{s 0} i+\alpha \cdot R_{s 0} i^{7}=r_{1} i+r_{7} i^{7}$.

Let us take $T_{0}=0.2 \times 10^{-10} ; V_{0}=I_{0} \approx 10^{-12} ; \mu=5 \times 10^{10}$; $\mu T_{0}=5 \times 10^{10} \times 0.2 \times 10^{-10}=1 ; \mathrm{e}^{-\mu T}=\mathrm{e}^{-5 \times 10^{10} \times 1.34 \times 10^{-8}}=\mathrm{e}^{-670} \approx 0 ; V_{0}=10^{-12}$. Then

$$
\begin{aligned}
& 2 \sqrt{L} /\left(G_{0} Z_{0}+1\right)+\mathrm{e}^{-670}\left|G_{0} Z_{0}-1\right| /\left(G_{0} Z_{0}+1\right) \Rightarrow 2 \sqrt{0.2 \times 10^{-6}} \leq(149 / 35)+1 ; \\
& 10^{4} \times \mathrm{e}^{-670}+\mathrm{e}\left(149+\left|r_{1}\right|+\left|r_{7}\right|\left(\mathrm{e}^{8}-1\right) /(\mathrm{e}-1)\left(10^{-12} / 7\left(2 \sqrt{0.2 \times 10^{-6}}\right)\right)^{7}\right) ; \\
& \leq 5 \times 10^{10} \times 0.2 \times 10^{-6}=10^{4}
\end{aligned}
$$$$
K_{V}=\mathrm{e}^{-670}|(149 / 35)-1| /((149 / 35)+1) \approx 0<1 ;
$$$$
K_{I} \approx \frac{2.72}{10^{4}}\left(149+\left|r_{1}\right|+\left|r_{7}\right| 637\left(10^{-9}\right)^{6}\right)<1 .
$$

The periodic solution is a particular case when $T_{0}=t_{k+1}-t_{k}(k=0,1,2, \cdots)$. We can choose an initial approximation $V^{(0)}(t)=V_{0} \sin \left(\omega_{0} t\right)$, $I^{(0)}(t)=I_{0} \cos \left(\omega_{0} t\right)$, where $\omega_{0}=2 \pi / T_{0}$. Then the first approximation is

$$
\begin{array}{r}
I^{(1)}(t)=\int_{k T_{0}}^{t} F_{V}\left(I^{(0)}, I^{(0)}\right)(s) \mathrm{d} s-\left(\left(t-k T_{0}\right) / T_{0}\right) \int_{k T_{0}}^{(k+1) T_{0}} F_{V}\left(I^{(0)}, I^{(0)}\right)(s) \mathrm{d} s, \\
t \in\left[k T_{0},(k+1) T_{0}\right],(k=0,1,2, \cdots)
\end{array}
$$




$$
\begin{array}{r}
V^{(1)}(t)=\left[2 \sqrt{L} J_{0}(t)+\left(G_{0} Z_{0}-1\right) V^{(0)}(t-T)\right] /\left(G_{0} Z_{0}+1\right), \\
t \in\left[k T_{0},(k+1) T_{0}\right],(k=0,1,2, \cdots)
\end{array}
$$

and so on.

\section{Conclusions}

1) It is proved that $V\left(t_{k}\right)=V\left(\Lambda, t_{k}\right)=0$ and $I\left(t_{k}\right)=I\left(0, t_{k}\right)=0$. But it must give a qualitative estimate of the solution of System (1), that is, $u(x, t)=V(x, t) /(2 \sqrt{C})-I(x, t) /(2 \sqrt{C})$, $i(x, t)=V(x, t) /(2 \sqrt{L})+I(x, t) /(2 \sqrt{L})$.

We note that every characteristic of the hyperbolic system with negative slope passing through the point $\left(0, t_{k}\right)$ has the equation $x=-v t+v t_{k}$. Therefore $0=I\left(t_{k}\right)=I\left(0, t_{k}\right)=I\left(\Lambda, t_{k}-T\right)$ and $I(x, t)=0$ along the straight line $x=-v t+v t_{k}$ between 0 and $\Lambda$. In a similar way every characteristic with positive slope passing through the point $\left(\Lambda, t_{k}\right)$ has the equation $x=v t-v t_{k}+\Lambda$ and then $0=V\left(t_{k}\right)=V\left(\Lambda, t_{k}\right)=V\left(0, t_{k}-T\right)$ and $V(x, t)=0$ along the straight line $x=v t-v t_{k}+\Lambda$ between 0 and $\Lambda$. In order to find common zeroes of $u(x, t)$ and $i(x, t)$ we have to intersect the straight lines $x=-v t+v t_{k}$ and $x=v t-v t_{k}+\Lambda$.

2) A general method for analysis of transmission lines terminated by polynomial nonlinearities is proposed and it is demonstrated on the 7-th order polynomial.

3) An explicit solution by successive approximations can be obtained.

\section{References}

[1] Scott, A. (1970) Active and Nonlinear Wave Propagation in Electronics. John Wiley \& Sons, New York.

[2] Nagumo, J. and Shimura, M. (1961) Self-Oscillation in a Transmission Line with a Tunnel Diode. Proceedings of the IRE, 49, 1281-1291. https://doi.org/10.1109/JRPROC.1961.287920

[3] Shimura, M. (1967) Analysis of Some Nonlinear Phenomena in a Transmission Line. IEEE Transactions on Circuit Theory, 14, 60-68. https://doi.org/10.1109/TCT.1967.1082648

[4] Chua, L.O., Desoer, C.A. and Kuh, E.S. (1987) Linear and Nonlinear Circuits. McGraw Hill Book Company, New York.

[5] Wadell, B.C. (1991) Transmission lines design Handbook. Artech House, Inc., Norwood, MA.

[6] Nguyen, C. (2001) Analysis Methods for RF, Microwave, and Millimeter-Wave Planar Transmission Line Structure. John Wiley \& Sons, New York.

[7] Misra, D.K. (2004) Radio-Frequency and Microwave Communication Circuits. John Wiley \& Sons, New York. https://doi.org/10.1002/0471653764

[8] Caloz, C. and Ito, T. (2006) Electromagnetic Metamaterials: Transmission Line Theory and Microwave Applications. John Wiley \& Sons, New York.

[9] Pozar, D.M. (2012) Microwave Engineering. John Wiley \& Sons, New York.

[10] Miano, G. and Maffucci, A. (2010) Transmission Lines and Lumped Circuits. 2nd 
Edition, Academic Press, New York.

[11] Martín, F. (2015) Artificial Transmission Lines for RF and Microwave Applications. Wiley Series in Microwave and Optical Engineering. https://doi.org/10.1002/9781119058403

[12] Singh, R. (2013) Circuit Theory and Transmission Lines. McGraw Hill Education.

[13] Makwana, V. and Bhalja, B. (2016) Transmission Line Protection Using Digital Technology. Energy Systems in Electrical Engineering, Springer.

[14] Ruikar, S. (2016) Electromagnetics and Transmission Lines. Nirali Prakashan.

[15] Lundquist, R.A. (2015) Transmission Line Construction: Methods and Costs. Forgotten Books.

[16] Kalaga, S. and Yenumula, P. (2016) Design of Electrical Transmission Lines: Structures and Foundations. CRC Press. https://doi.org/10.1201/9781315755687

[17] Angelov, V.G. (2014) A Method for Analysis of Transmission Lines Terminated by Nonlinear Loads. Nova Science, New York.

[18] Maas, S.A. (2003) Nonlinear Microwave and RF Circuits. Artech House, Boston, London.

[19] Kollberg, E.L., et al. (1992) Current Saturation in Submillimeter-Wave Varactors. IEEE Transaction, 40, 831. https://doi.org/10.1109/22.137387

Submit or recommend next manuscript to OALib Journal and we will provide best service for you:

- Publication frequency: Monthly

- 9 subject areas of science, technology and medicine

- Fair and rigorous peer-review system

- Fast publication process

- Article promotion in various social networking sites (LinkedIn, Facebook, Twitter, etc.)

- Maximum dissemination of your research work

Submit Your Paper Online: Click Here to Submit

Or Contact service@oalib.com 